\title{
Rapid non-invasive genotyping of reporter transgenic mammals
}

\author{
Wiebke Garrels, Nicole Cleve, Heiner Niemann, Wilfried A. Kues \\ Friedrich-Loeffler-Institut, Institut für Nutztiergenetik, Mariensee, Germany
}

Here we describe a non-invasive method for rapid and highly reproducible genotyping of transgenic mammals with ubiquitous expression of fluorophore reporters. Hair samples from transgenic mice and pigs with systemic expression of the fluorophore reporter Venus were analyzed with a fluorescence microscope in few minutes. The hair samples can be preserved for long-term storage at ambient temperature conditions. This non-invasive method is useful for genotyping of transgenic large animals and contributes to animal welfare by reducing stress and discomfort of the animals during sample collection.

BioTechniques 52:1-4 (May 2012) doi 10.2144/000113874

Keywords: Sleeping Beauty transposon; fluorescent protein; phenotype; animal welfare; expression; ubiquitous; keratin; cornified keratinocyte; extracellular

Genetic engineering of large mammals is critical for the development of improved biomedical models, pharmaceutical research, and implementation of novel traits in animal breeding $(1,2)$. Recently, the generation of transgenic pigs with systemic expression of vital fluorophore markers has found widespread interest (3-11), e.g., for improving gene transfer technologies in pigs $(3,5,7-9$, $11)$, for transplantation studies $(4,10)$, for testing the functionality of multicistronic transgene constructs (2), and for visualizing epigenetic phenomena during spermatogenesis and fertilization (6).

Southern blotting and PCR are routinely used for genotyping transgenic founders or screening their offspring. Both methods rely on genomic DNA isolated from ear or blood samples. Sampling of ear biopsies or blood is associated with stress and discomfort for the animals. Expression of transgenic fluorophore markers can be visualized by specific excitation light in the skin of live animals, but conditions in most animal quarters do not provide lighting for quantitative fluorimetric visualization. Here, we developed and validated a non-invasive method based on quantitative measurement of fluorescence intensities of hair samples. This method is compatible with rapid and reproducible genotyping, and does not require removal of hair roots (12). Hair sampling causes minimal to no stress to the animals. Processing and evaluation of the hair samples can be done in less than one hour (compared with one day for PCR or 2-3 days for a Southern blot).

As a proof-of-principle, hair samples were collected from an established Venus transgenic mouse line generated by cytoplasmic injection of transposon plasmids into zygotes $(13,14)$. [A nimals were maintained and handled according to the German laws for animal welfare and the German law regarding genetically modified organisms. Experiments were approved by an external ethics committee (Niedersächsisches Landesamt für Verbraucherschutz und Lebensmittelsicherheit, AZ 33.9-4250204-09/1718).]

Venus is an improved variant of the enhanced yellow fluorescent protein (EYFP), and is characterized by faster maturation at $37^{\circ} \mathrm{C}$, increased tolerance to low $\mathrm{pH}$ conditions, improved refolding after denaturation, and stronger relative fluorescence (15). The monomeric Venus transposon integration was transmitted through the germ line according to Mendelian rules. Breeding of hemizygous transgenic animals resulted in wildtype, hemizygous, and homozygous offspring in the expected ratio of $1: 2: 1$ (14). Samples of 10-50 hairs were collected from littermates with the three different genotypes. The hair samples were placed side by side under a stereomicroscope equipped with epifluorescence optics (Olympus SZX16, Hamburg, Germany), a SDF PLAPO 1.6x PFX objective, and a high resolution digital camera (Olympus DP72). Fluorescence images (excitation: $450-490 \mathrm{~nm}$, emission: 515-550 nm, dichroic mirror: $505 \mathrm{~nm}$ ) were taken under normalized conditions (500 ms exposure). Fluorescence images (Figure 1) were analyzed with the aid of CellF software (Olympus). A horizontal histogram function of CellF was employed for quantitative analysis of fluorescence intensity (Figure 1). Fluorescence levels in the hair samples correlated with the wildtype, hemizygous, or

\section{Method summary:}

Here we report a non-invasive method to genotype transgenic mammals expressing fluorophore markers using fluorescence microscopy on hair samples. Quantitative assessment is also possible using this technique, enabling rapid differentiation of hemizygous and homozygous status. Fluorescence properties of the Venus fluorophore, incorporated into mammalian hair, were fully maintained during long-term storage. 
homozygous genotypes (Figure 1; two replicates; $\mathrm{n}=6$ ).

Presumably, Venus protein is produced in keratinocytes and the protein is deposited in the proliferative zone (Figure 2) of the hair follicle during terminal differentiation of keratinocytes to cornified keratinocytes.

Next, the suitability of this non-invasive method for the analysis of hair from transgenic pigs was assessed. Hair samples were collected from littermates of the F1 and F2 generations, and of cloned Venus-transposon transgenic animals, (5) and analyzed as described above (Figure 2 A-C). A direct positive correlation of the fluorescence intensity in porcine hair and the respective genotype was determined by Southern blotting (Figure 2). In three replicates a total of 31 hair samples were analyzed. Ideally, hair samples should be collected from littermates or age-matched animals. Fluorescence intensity of porcine hair showed a greater variation than those of their murine counterparts, most likely due to greater variation in diameter. Within longitudinal sections only minor variations of fluorescence intensity were found (Figure 2D).

When porcine hair samples were exposed for extended periods to maximum specific excitation (fluorescence bulb: EXFO; X-Cite Series 120Q, $100 \mathrm{~W})$, Venus fluorescence intensity was reduced by only $25-30 \%$ over a period of $4 \mathrm{~h}$ (Figure 2E; 3 replicates, $\mathrm{n}=18$ ). Over a storage period of three month at room temperature in the dark, Venus fluorescence was maintained in transgenic hair at the same intensity without the addition of any preservatives (not shown). Thus long-term preservation and re-analysis of hairs is possible at different time points after sampling. To directly confirm the presence of Venus protein, hair samples were extracted and separated by SDS-PAGE (16). Western blotting with a polyclonal anti-EGFP antibody (Fisher Scientific, Schwerte, Germany) revealed a specific $\sim 30 \mathrm{kD}$ protein band indicating the Venus protein in extracts of transgenic hair samples (Figure 2F). Venus and EGFP are $97 \%$ identical at the amino acid sequence.

In summary, we report a rapid non-invasive method for genotyping that complements and extends the available molecular methods for genotyping. We showed that transgenic mammals with ubiquitous Venus expression can be discriminated from wildtype animals and that a quantitative assessment of hemizygous versus homozygous status
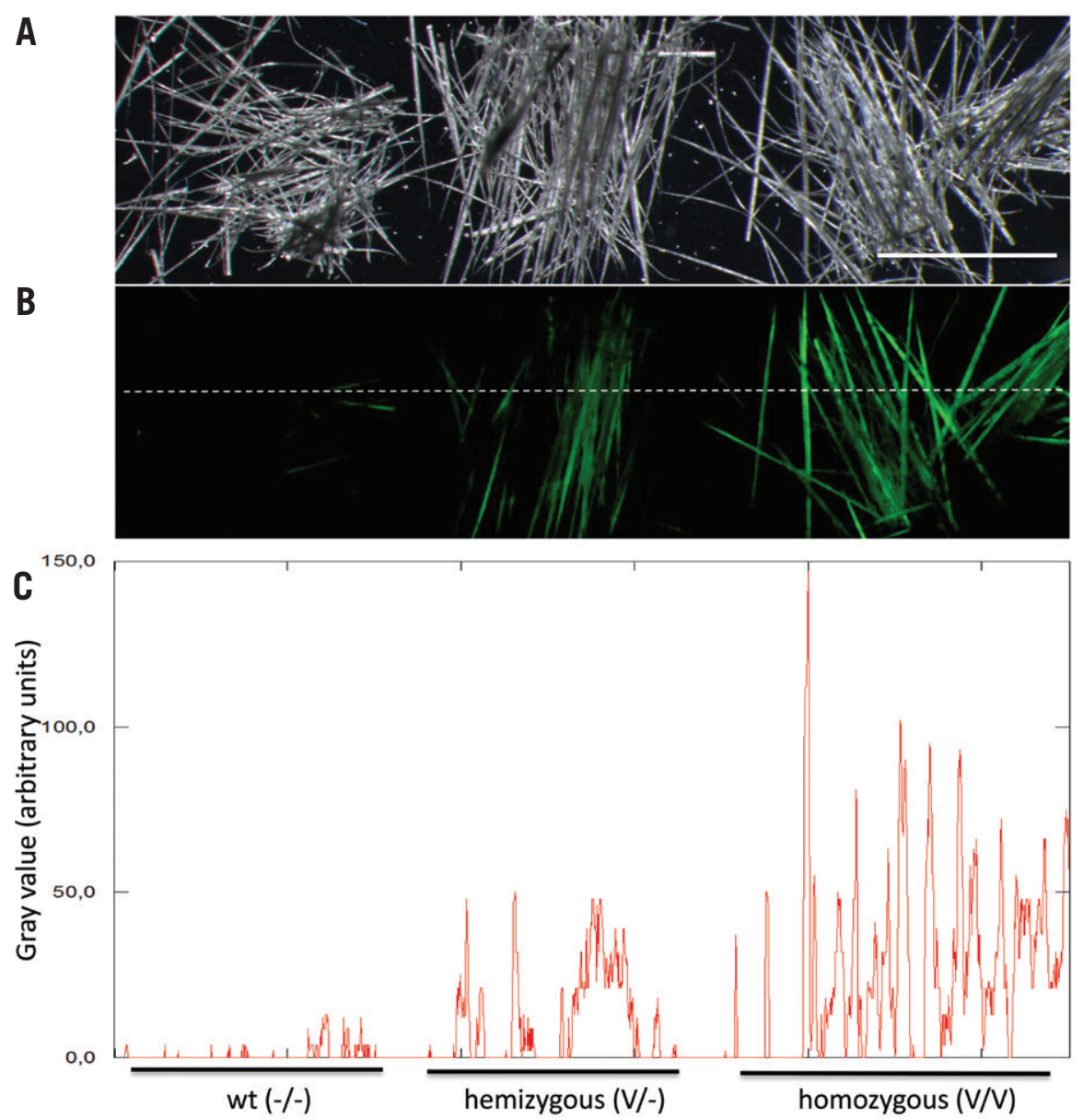

Figure 1. Non-invasive genotyping of mouse hair from littermates of hemizygous parents. (A) Darkfield view of fur samples from three littermates representing wildtype, hemizygous, and homozygous status of the Venus transposon (2 replicates, $n=6$ ). Bar $=2000 \mu \mathrm{m}$. (B) Specific Venus-fluorescence of the hair samples in A, dotted line indicates measured area for intensity histogram. (C) Fluorescence intensities of the hair samples. The known genotypes are indicated. Note that the fluorescence image was captured within the dynamic range of the samples and was not optimized for aesthetic appearance. For intensity measurement, a gray value image was used. V, Venus transposon allele; no Venus allele.

is possible. In more complex transgenic scenarios, such as concatemeric insertions or integrations in differently permissive loci, hair genotyping may reach its limits. The Venus protein was found to be deposited in mammalian hair and, importantly, the fluorescent properties seemed to be fully maintained in this extracellular environment. Major advantages of hair genotyping are its applicability to field conditions and the possibility for long-term sample preservation at low costs. The method allows screening of large sample numbers in a medium-throughput manner. Repetitive sampling and analyses are possible, enabling assessment of the effects of environmental conditions, feeding, or aging on transgene expression. Hair analysis might be useful for assessing conditional transgene regulation in transgenic large mammals bearing promoter constructs, which are responsive to exogenously supplied small molecules, such as tetracycline derivatives $(17,18)$. Of course, this does require additional genetic modifications. Farm animals easily get used to hair sampling, which avoids injuries and discomfort. It is assumed that this methodology can be translated to transgenic models in other mammalian species $(17,19)$ and possibly to feathers of transgenic birds.

\section{Acknowledgments}

The expert technical support by Stephanie Holler, Brigitte Barg-Kues, 
A
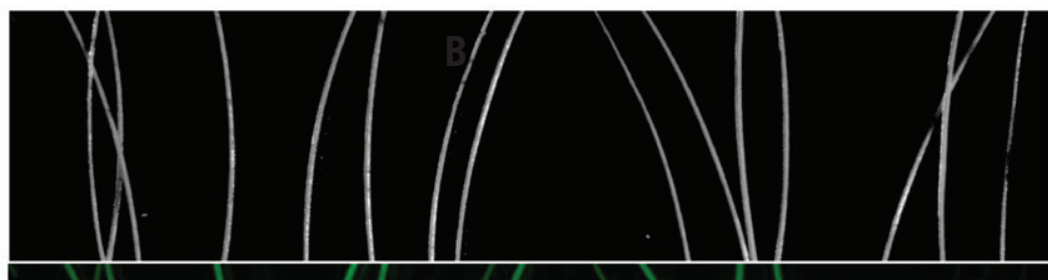

B

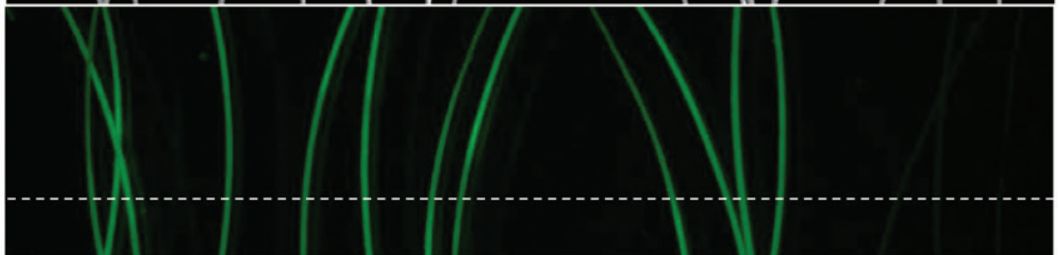

C

C 120,0

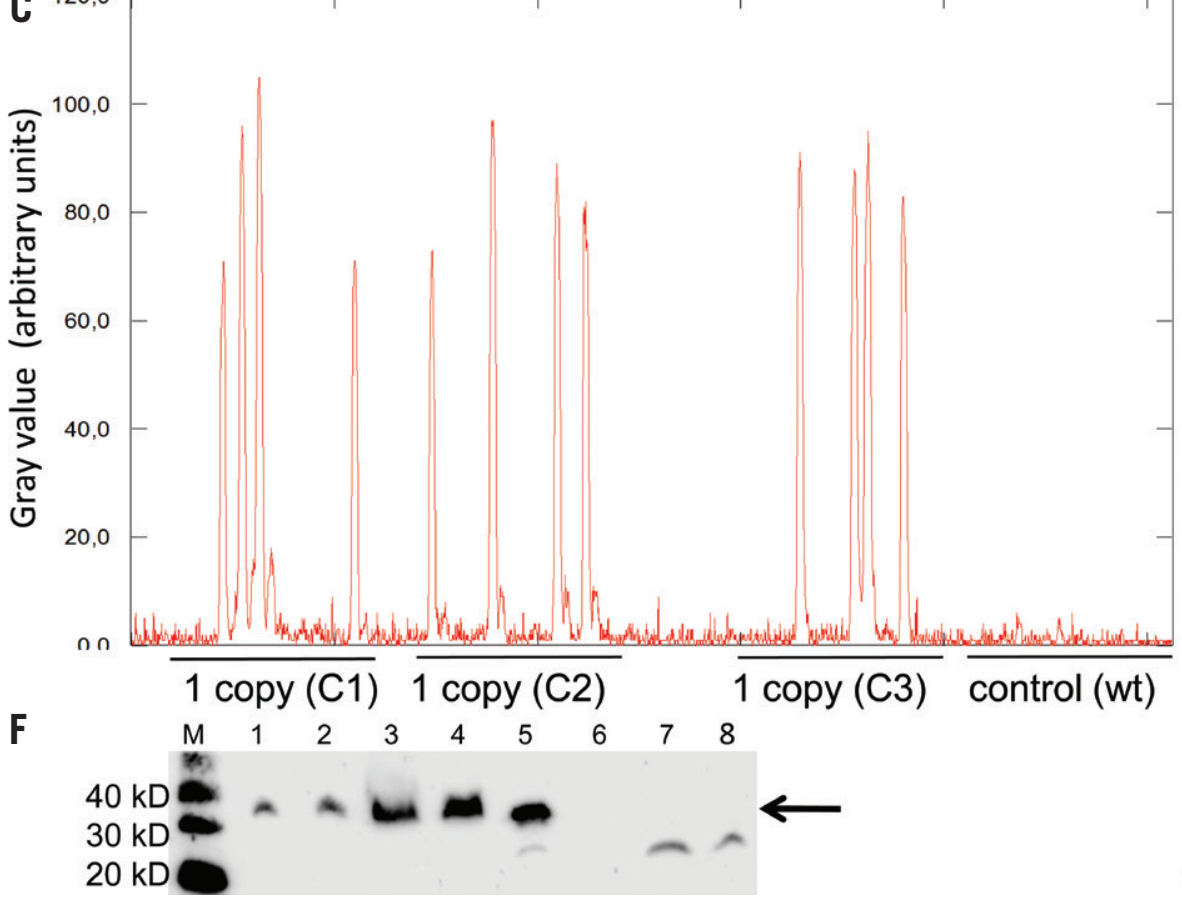

D
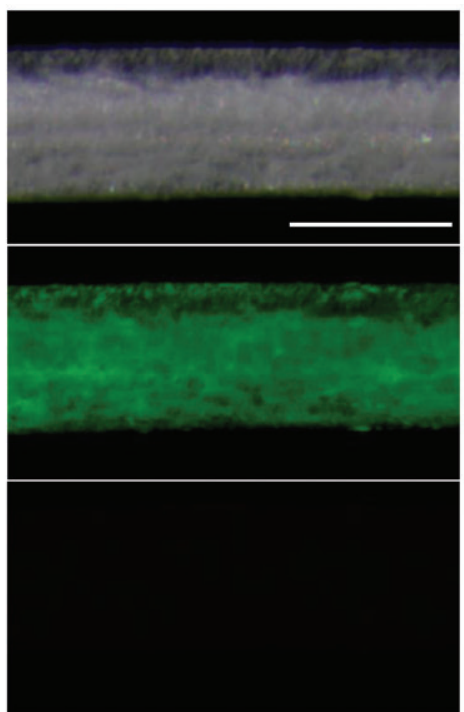

E
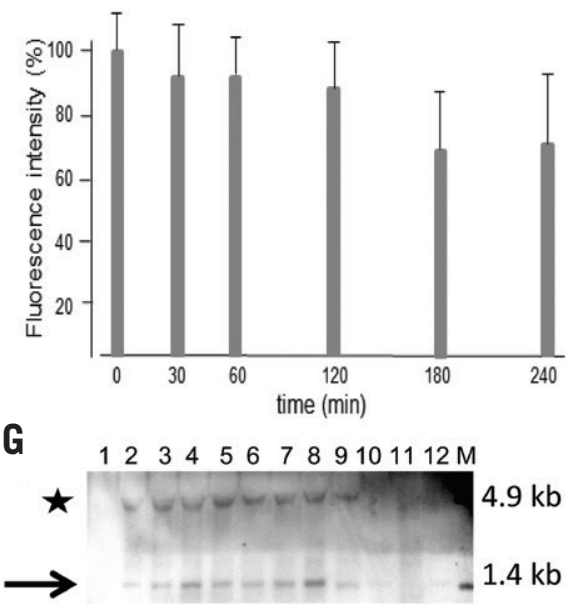

Figure 2. Non-invasive genotyping of hair samples from transposon transgenic piglets. (A) Darkfield view of hair from 4 piglets ( 3 replicates, total $n=31$ ). (B) Specific Venus-fluorescence of the hair in A, dotted line indicates measured area. (C) Fluorescence intensities of the hair samples. The deduced numbers of Venus transposon copies are indicated. Venus positive samples were collected from three cloned piglets (C1-C3, corresponding to lanes 2,3,4 in Figure 2G), carrying a single (monomeric) Venus transposon. The control hair sample was collected from a non-transgenic age-matched animal. (D) Longitudinal section of a Venus-transgenic porcine hair (C1) at high resolution, displayed under darkfield conditions, as well as under specific Venus excitation and specific mCherry excitation (negative control). Bar $=100 \mu \mathrm{m}$. (E) Stability of specific Venus fluorescence in pig hair during continuous longterm excitation ( 3 replicates, $n=18$ ). Note the minimal bleaching during a $4 \mathrm{~h}$ excitation. ( $F$ ) Western blot detection of Venus protein in hair extracts. $M$, molecular size marker; 1, 2 delipidized hair (C1 and C2) was extracted with thiourea, urea, and 2-mercaptoethanol (16); 3, 4, hair extracts (C1 and C2) were desalted by dialysis and concentrated with AmiconUltra (Millipore, Schwalbach, Germany; 5, lymphocytes of transgenic pig (C1); 6, control (hair from wildtype); 7,8, controls (lymphocytes of two wildtype animals). Arrow indicates the Venus protein. In lymphocyte extracts a weak non-specific signal with a molecular weight of about $25 \mathrm{kD}$ is visible. (G) Southern blot genotyping of animals used for hair analysis. A litter of 8 cloned piglets was genotyped by standard blotting procedures. According to the restriction of genomic DNA, an internal fragment of $\sim 1.4 \mathrm{~kb}$ (arrow) and an external fragment (asterisk) of $>3 \mathrm{~kb}$ are predictive for hemizygous cloned animals carrying a monomeric integration [for details see (6)]. 1, carrier sow (non-transgenic); 2-9, cloned piglets; 10, empty; 11, non-related wildtype animal; 12, empty; M, positive control (with bands of 1.4 and $4.9 \mathrm{~kb}$ ).

Maren Ziegler and Karin Klingemann is gratefully acknowledged. Katharina Katter and Thomas Rülicke (Veterinary University Vienna, Austria) shared unpublished data on Sleeping Beauty transgenesis in mice. Financial support by DFG is acknowledged (KU 1586/2-1).

\section{Competing interests}

The authors declare no competing interests.

\section{References}

1. Kues, W.A. and H. Niemann. 2011. Advances in farm animal transgenesis. Prev. Vet. Med. 102:146-156.
2. Whyte, J.J. and R.S. Prather. 2011. Genetic modifications of pigs for medicine and agriculture. Mol. Reprod. Dev. 78:879-891.

3. Brunetti, D., A. Perota, I. Lagutina, S. Colleoni, R. Duchi, F. Calabrese, M. Seveso, E. Cozzi, et al. 2008. Transgene expression of green fluorescent protein and germ line transmission in cloned pigs derived from in vitro transfected adult fibroblasts. Cloning Stem Cells 10:409-419. 
4. Kawarasaki, T., K. Uchiyama, A. Hirao, S. Azuma, M. Otake, M. Shibata, S. Tsuchiya, S. Enosawa, et al. 2009. Profile of new green fluorescent protein transgenic Jinhua pigs as an imaging source. J. Biomed. Opt. 14:054017.

5. Garrels, W., L. Mátés, S. Holler, A. Dalda, U. Taylor, B. Petersen, H. Niemann, Z. Izsvák, et al. 2011. Germline transgenic pigs by Sleeping Beauty transposition in porcine zygotes and targeted integration in the porcine genome. PLoS One 6:e23573.

6. Garrels, W., S. Holler, U. Taylor, D. Herrmann, C. Struckmann, S. Klein, B. Barg-Kues, M. Nowak-Imialek, et al. 2011. Genotype-independent transmission of transgenic fluorophore protein by boar spermatozoa. PLoS One 6:e27563.

7. Carlson, D.F., J.R. Garbe, W. Tan, M.J. Martin, J.R. Dobrinsky, P.B. Hackett, K.J. Clark, and S.C. Fahrenkrug. 2011. Strategies for selection marker-free swine transgenesis using the Sleeping Beauty transposon system. Transgenic Res. 20:1125-1137

8. Jakobsen, J.E., J. Li, P.M. Kragh, B. Moldt, L. Lin, Y. Liu, M. Schmidt, K.D. Winther, et al. 2011. Pig transgenesis by Sleeping Beauty DNA transposition. Transgenic Res. 20:533-545.

9. Matsunari, H., M. Onodera, N. Tada, H. Mochizuki, S. Karasawa, E. Haruyama, N. Nakayama, H. Saito, et al. 2008. Transgenic-cloned pigs systemically expressing red fluorescent protein, Kusabira-Orange. Cloning Stem Cells 10:313-323.

10. Hsiao, F.S.H., W.S. Lian, S.P. Lin, C.J. Lin, Y.S. Lin, E.C.H. Cheng, C.W. Liu, E.C.H. Cheng, et al. 2011. Toward an ideal animal model to trace donor cell fates after stem cell therapy: Production of stably labeled multipotent mesenchymal stem cells from bone marrow of transgenic pigs harboring enhanced green fluorescence protein gene. J. Anim. Sci. 89:3460-3472.

11. Garrels, W. Ivics, Z. and Kues, W.A. 2012 Precision genetic engineering in large mammals. Trend. Biotechnol. (epub ahead of print Apr. 19.) http://dx.doi.org/10.1016/j. tibtech.2012.03.008

12. Terrell, K.A., T.A. Rassmussen, C. Trygg, B.A. Bunnell, and W.R. Buck. 2007. Molecular beacon genotyping from hair roots in the twitcher mouse and Rhesus macaque. J. Neurosci. Methods 163:60-66.

13. Iqbal, K., B. Barg-Kues, S. Broll, J. Bode, H Niemann, and W.A. Kues. 2009. Cytoplasmic injection of circular plasmids allows targeted expression in mammalian embryos. Biotechniques 47:959-968.

14. Garrels, W., H. Niemann, and W.A. Kues. 2012. Mendelian inheritance by eye. Mol. Reprod. Dev. 79:75.

15. Nagai, T., K. Ibata, E.S. Park, M. Kubota, K. Mikoshiba, and A. Miyawaki. 2002. A variant of yellow fluorescent protein with fast and efficient maturation for cell-biological applications. Nat. Biotechnol. 20:87-90.

16. Nakamura, A., M. Arimoto, K. Takeuchi, and T. Fuji. 2002. A rapid extraction procedure of human hair proteins and identification of phosphorylated species. Biol. Pharm. Bull. 25:569-572.

17. Kim, M.J., H.J. Oh, J.E. Park, G.A. Kim, S.G. Hong, G. Jang, M.S. Kwon, B.C. Koo, et al. 2011. Generation of transgenic dogs that conditionally express green fluorescent protein.
Genesis 49:472-478.

18. Kues, W.A., R. Schwinzer, D. Wirth, E. Verhoeyen, E. Lemme, D. Herrmann, B. Barg-Kues, H. Hauser, et al. 2006. Epigenetic silencing and tissue independent expression of a novel tetracycline inducible system in doubletransgenic pigs. FASEB J. 20:1200-1202.

19. Hiripi, L., D. Negre, F.L. Cosset, K. Kvell, T. Czömpöly, M. Baranyi, E. Gócza, O. Hoffmann, et al. 2010. Transgenic rabbit production with simian immunodeficiency virus-derived lentiviral vector. Transgenic Res. 19:799-808.

Received 25 April 2012; accepted 04 May 2012.

Address correspondence to Wilfried A. Kues, Friedrich-Loeffler-Institut, Institut für Nutztiergenetik, Mariensee, 31535 Neustadt, Germany.Email:wilfried.kues@fli.bund.de

To purchase reprints of this article, contact: biotechniques@fosterprinting.com 